\title{
An Integrated Method of Multiradar Quantitative Precipitation Estimation Based on Cloud Classification and Dynamic Error Analysis
}

\author{
Yong Huang, ${ }^{1,2}$ Huijuan Liu, ${ }^{1}$ Yun Yao, ${ }^{1}$ Ting Ni, $^{2}$ and Yan Feng ${ }^{1}$ \\ ${ }^{1}$ Anhui Meteorology Institute, Anhui Key Lab of Atmospheric Science and Satellite Remote Sensing, Hefei, Anhui 230031, China \\ ${ }^{2}$ Shouxian National Climate Observation Station, Huainan, Anhui 232200, China \\ Correspondence should be addressed to Yong Huang; hy121_2000@126.com
}

Received 17 November 2016; Accepted 15 February 2017; Published 5 March 2017

Academic Editor: Zhe Li

Copyright (C) 2017 Yong Huang et al. This is an open access article distributed under the Creative Commons Attribution License, which permits unrestricted use, distribution, and reproduction in any medium, provided the original work is properly cited.

Relationships between radar reflectivity factor and rainfall are different in various precipitation cloud systems. In this study, the cloud systems are firstly classified into five categories with radar and satellite data to improve radar quantitative precipitation estimation (QPE) algorithm. Secondly, the errors of multiradar QPE algorithms are assumed to be different in convective and stratiform clouds. The QPE data are then derived with methods of $Z-R$, Kalman filter (KF), optimum interpolation (OI), Kalman filter plus optimum interpolation (KFOI), and average calibration (AC) based on error analysis on the Huaihe River Basin. In the case of flood on the early of July 2007, the KFOI is applied to obtain the QPE product. Applications show that the KFOI can improve precision of estimating precipitation for multiple precipitation types.

\section{Introduction}

With operational running of the China New Generation Weather Radar in mainland China, a lot of studies have been reported with focus on radar quantitative precipitation estimation (QPE) in China [1-6]. QPE algorithms in these studies include the relationship between the radar echo and the observed precipitation, precipitation estimation using merged radar and rain gauge data, integrated approach of multiradar precipitation estimation methods, and QPE for specific region.

In the end of the 1940s, Marshall et al. [7] proposed the Z$R$ model to estimate rainfall intensity with radar reflectivity factor. The $Z-R$ relation is a precipitation estimation method based on the relationship between the radar reflectivity factor $(Z)$ and rainfall intensity $(R)$. The merging methods actually start from $Z-R$ relation estimation procedure but have additional correction procedures, such as $\mathrm{Hu}$ et al. [8] using Kalman filter to determine the coefficients of the variation equation and calibrating precipitation; more details can be seen in [3, 8-12]. For the operational monitoring of rainfall, the widely used radar precipitation estimation methods include $Z$ (radar reflectivity factor)- $R$ (rainfall intensity) relationship method [7] and radar-gauge merging methods, such as average calibration (AC) method [10], Kalman filter (KF) method $[8,13]$, optimal interpolation (OI) method [14, 15], and integrated Kalman filter and optimal interpolation (KFOI) [9].

In recent years, several kinds of algorithms have been developed to improve the accuracy of radar QPE. There algorithms include the merging approaches based on radar observations and the approaches based on vertical profiles of reflectivity identification and enhancement (VPR-IE) $[16,17]$.

Besides, a feasible approach is to merge different kinds of observations to improve the precision of radar precipitation estimation. Previous studies showed that more accurate precipitation estimate can be obtained with application of the integrated methods $[6,18,19]$. The results of Guan's study show that the accuracy of rainfall estimation can be improved based on the combination of different approach with Principal Component Analysis (PCA) method. PCA is a multidimensional orthogonal linear transformation based 
on Karhunen-Loève transform, which is also named K-L transform integrated method (KL) [19]. As shown in the results of Huang et al. [18], the accuracy of QPE can be improved by using the integrate technique, which is to calculate the variation of QPE errors in different areas and select the optimal one with minimum error variation.

The objective of this study is to improve the accuracy of previous integrated method based on error analysis in fixed boundary of regions in Huaihe River Basin (HRB) [18]. The previous error analysis method is thus also named as Fixed Errors Analysis (FEA). According to FEA, the whole HRB has been classified into 15 areas with fixed boundary for errors statistic. The areas of errors analysis are fixed and do not vary with the change of precipitation system in these 15 areas. Since the rainfall is related to the precipitating cloud system and not related to the boundary of river basin or boundaries of administrative areas, the errors of QPEs are only calculated in fixed 15 areas in previous error analysis. In addition, the QPE errors of whole rainfall system could not be evaluated. To overcome the shortcoming of error analysis in fixed areas FEA, satellite and radar data are applied to classify cloud system. Then the optimal QPE method is selected from five methods by assessing the variation of the random errors from latest precipitation estimates for different precipitation cloud systems. Because the regions of error analysis are dynamically changing with the cloud system, the method in this study is named as Dynamic Errors Analysis (DEA).

\section{Dynamic Errors Analysis Integrated Method}

For a specific precipitation type, application of the $Z-R$ relation method and four joint radar-gauge correction methods can generate different estimation errors. By comparing the precipitation estimates with the observations from rain gauges, the best method can be selected for further bias correction. The selected method with bias correction is expected to generate more accurate precipitation estimates for future rainfall.

2.1. Assumptions. There are two assumptions in this method. One is about estimation error; the other is related to precipitation systems. First, we assume that the errors of radar QPE are composed of systematic bias and random errors. Second, we assume that the errors of radar QPE vary with the transformation of precipitation system. There is an optimal QPE method with the minimum random error for a precipitation system among these methods.

The systemic bias is a constant, while the random error varies with the different algorithms and the evolution rainfall cloud system. The better algorithm is the small random error, and vice versa. The error of rainfall can be evaluated by analyzing the selected time series of the random errors.

The rainfall estimation error is defined as $W(t)=W_{s}+$ $\widetilde{W}(t)$, where $W_{s}$ is systematic bias, and it is a constant. $\widetilde{W}(t)$ represents random error and it varies with time. The estimation error of a specific period can be calculated by

$$
W(t)=R_{g}(t)-R_{r}(t) .
$$

The systematic bias $W_{s}$ is assumed as the average of errors in a specific period.

$$
\begin{aligned}
W_{s} & =\frac{1}{T} \times \sum_{t=1}^{T}\left(R_{g}(t)-R_{r}(t)\right) \\
\widetilde{W}(t) & =\left(R_{g}(t)-R_{r}(t)\right)-W_{s},
\end{aligned}
$$

where $R_{g}(t)$ is observed precipitation at $t$ time by ground gauge and $R_{r}(t)$ is estimated precipitation at $t$ time by radar.

The variance of estimation error $\left(S_{W}\right)$ is applied to evaluate the stability of errors as the following formula:

$$
\begin{aligned}
S_{W} & =\frac{1}{T} \times \sum_{t=1}^{T}(W(t)-\bar{W})^{2}=\frac{1}{T} \times \sum_{t=1}^{T}\left(W(t)-W_{s}\right)^{2} \\
& =\frac{1}{T} \times \sum_{t=1}^{T} \widetilde{W}(t)^{2} .
\end{aligned}
$$

According to (3), the variance of observation errors is actually also the value of random errors. Therefore, the value of the random errors during a specific period can be obtained by evaluating the variance of the estimation errors during that period, so the ability of estimation can be acquired.

For the precipitation systems, it is assumed that there are different rainfall patterns (warm convective precipitation, cold convective precipitation, warm stratiform precipitation, cold stratiform precipitation, and mixed precipitation), and the abilities of the estimation method for different patterns are various, because the rain drop size distribution (DSD) is different for several patterns. The ability of estimation for a method can be evaluated by analyzing the variance of the estimation errors. Small variance indicates small random errors and a stable time series of estimation errors, suggesting that the estimation method is good, and vice versa.

Based on the assumptions and analysis of the estimation error time series, the optimal method is screened out from several QPE methods.

2.2. Integrated Method. The $Z-R$ relation varies with precipitation types. Each estimation method has its preferred precipitation pattern. To improve the efficiency of the integrated method, we first determine the cloud pattern according to satellite brightness temperature and radar echo info and then apply different $Z-R$ relationship on different precipitation pattern. The detailed procedures include the following.

Step 1. Determine cloud type by analyzing satellite brightness temperature and radar reflectivity factor database using threshold technique based on satellite cloud classification method [20]. To improve the rainfall estimation, this threshold-based techniques is revised using new experiential radar reflectivity $(R)$ thresholds; that is, $R>15 \mathrm{Dbz}$ for stratiform rainfall, $R>30 \mathrm{Dbz}$ for convective rainfall, and $R>50 \mathrm{dBZ}$ for deep convective rainfall. In addition, the satellite infrared temperature, coupled with radar reflectivity, is used to classify the cloud into 5 categories. The specific thresholds can be seen in Table 1 . 
TABLE 1: Thresholds of cloud type Determined.

\begin{tabular}{llc}
\hline & Thresholds & $Z-I$ relationship \\
\hline Warm stratiform precipitation & $15 \mathrm{dBZ}<R<35 \mathrm{dBZ}$ and $T>267 \mathrm{~K}$ & $Z=131 I^{1.44}$ \\
Cold stratiform precipitation & $15 \mathrm{dBZ}<R<35 \mathrm{dBZ}$ and $241 \mathrm{~K}<T \leq$ & $Z=200 I^{1.6}$ \\
& $267 \mathrm{~K}$ & \\
Mixed precipitation & $15 \mathrm{dBZ}<R<35 \mathrm{dBZ}$ and $221 \mathrm{~K}<T \leq$ & $Z 200 I^{1.35}$ \\
Warm convective precipitation & $241 \mathrm{~K}$ & $Z=300 I^{1.4}$ \\
Cold convective precipitation & $35 \mathrm{dBZ} \leq R<50 \mathrm{dBZ}$ and $T>267 \mathrm{~K}$ & $Z=403 I^{1.29}$
\end{tabular}
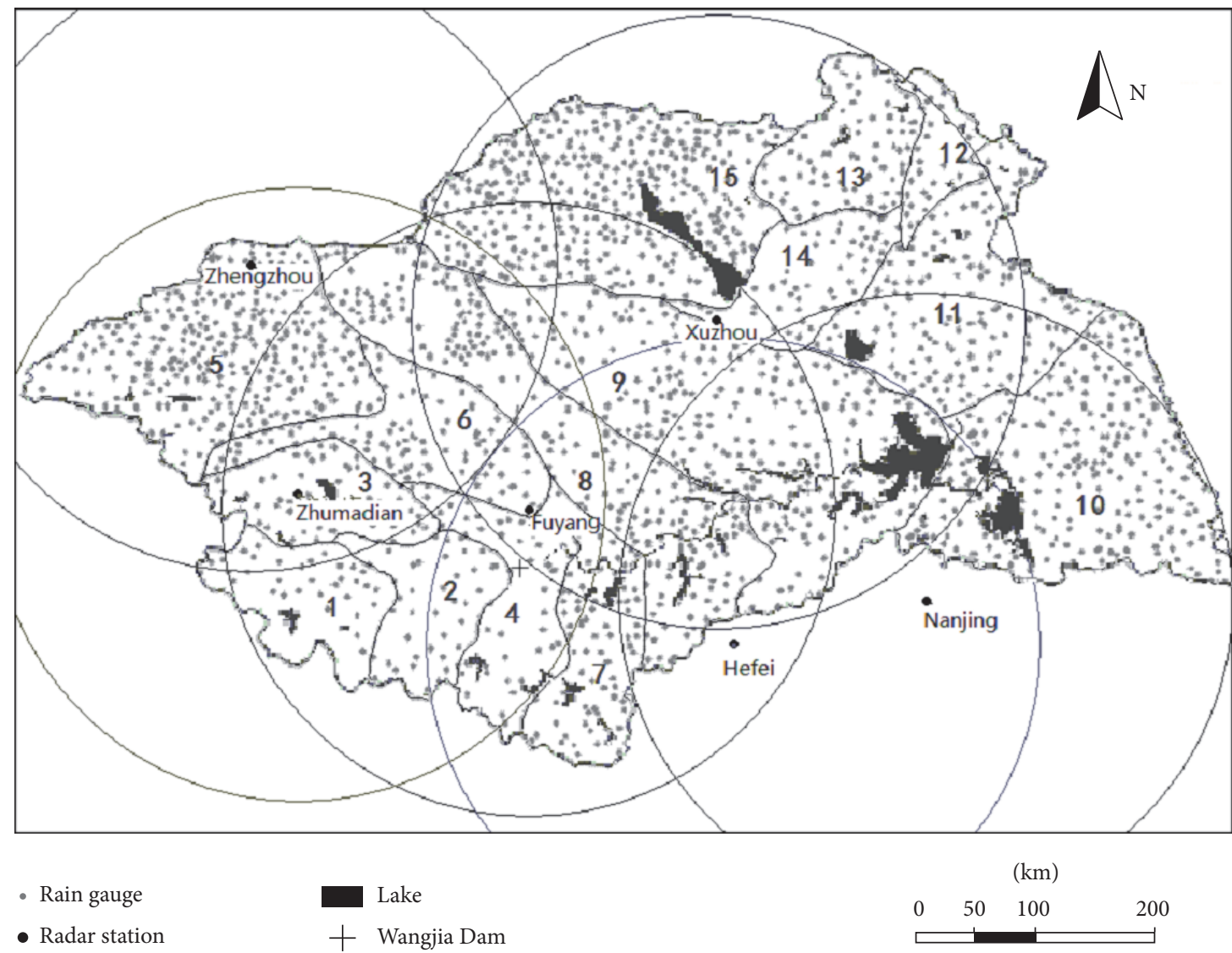

FIGURE 1: Distribution of the radars and rain gauges in Huaihe River Basin.

Step 2. Compute rainfall with 6-minute interval radar data from six radars (Hefei, Nanjing, Zhengzhou, Fuyang, Zhumadian, and Xuzhou radar) in HRB (Figure 1). The popular $Z-R$ relationships of stratiform and convective rainfall [21, 22] are applied to compute the cold stratiform and warm convective precipitation. For the other 3 cloud types, the drop size distribution (DSD) data in Huainan weather station from 2009 to 2012 are collected to compute the mode of $Z-R$ relationship with regression method.

Step 3. Correct the radar quality precipitation using ground gauge observations (Figure 1) with four correction methods (AC, KF, OI, and KFOI).
Step 4. With the results of cloud type, determine precipitation pattern, and errors of five QPE methods ( $Z-R$ relationship and four correction methods) for five rainfall patterns are calculated in the latest one hour (10 times QPE data with 6-minute interval). The most best QPE method is the one with the smallest variance of the estimation errors in the past.

Step 5. Correct system bias for QPE data of the best method. The system bias is calculated by averaging the error of radar QPE and rain gauge data in the latest one hour (10 times QPE data with 6-minute interval). Then subtract the system bias from the selected radar QPE data. 
TABLE 2: Relative errors and correlation coefficients of QPE and rain gauge from June 30 to July 9, 2007.

\begin{tabular}{lcccc}
\hline & \multicolumn{2}{c}{ Relative error } & \multicolumn{2}{c}{ Correlation coefficients } \\
& All cases & $>10 \mathrm{~mm} / \mathrm{h}$ & All cases & 0.86 \\
DEA & 0.33 & 0.26 & 0.87 & 0.65 \\
FEA & 0.34 & 0.32 & 0.82 & 0.68 \\
KL & 0.41 & 0.30 & 0.81 & 0.66 \\
AC & 0.42 & 0.3 & 0.81 & 0.66 \\
KF & 0.42 & 0.3 & 0.82 & 0.68 \\
OI & 0.41 & 0.28 & 0.82 & 0.68 \\
KFOI & 0.41 & 0.28 & 0.70 & 0.47 \\
$Z-R$ & 0.45 & 0.61 & & \\
\hline
\end{tabular}

\section{Verification}

During June 30 to July 9, 2007, due to the stable west Pacific subtropical high pressure, strong monsoon surge, dry cold air from middle-high latitudes, and propagating eastward convection disturbance over the Tibetan Plateau, a Meiyu front cloud system with heavy rainfall takes shape over HRB [23]. It is noted that a belt of heavy rainfall is over HRB. The HRB experienced several heavy rainfall storm cases successively, which lead to the greatest flood over HRB since 1954.

The successive heavy rainfall storms lead to two peak discharges passing the main hydrologic station (Wangjia Dam) in the main channel of Huaihe River. A major flood event was recorded in the downstream Huaihe River lower the Runheji hydrologic station. And flood events were recorded for most of the tributaries. The heavy rainfall event includes three stages: June 30-July 2, July 4-July 5, and July 8-July 9. The most intense precipitation occurred in the last stage, during which new records of maximum daily precipitation were recorded for several stations. The strong rainfall eventually leads to flood diversion in Wangjia Dam.

To estimate the precipitation, observations from six radars and surface rain gauges (Figure 1) are used to demonstrate the integrated method of precipitation estimation. And the $Z-R$ relationship method and four rain gauge correction methods (AC, KF, OI, and KFOI) of QPE are applied in heavy rain period from June 30 to July 9, 2007.

Figure 2 shows the precipitation estimation of middle stage of heavy rainfall case at 17:00-18:00 July 52007 (UCT) with $Z-R$ method, four correction methods, three integrated methods mentioned in Section 2, and the observations from rain gauge (using the Kriging interpolation technique of the Golden Software Surfer 8).

The results from estimates and observations are shown in graded color shading in Figure 2. According to the rain gauge observations, there is one major rainfall belt from west to east. The maximum rainfall area (MRA1) is located in the middle of the HRB. The second strong rainfall area (MRA 2) is located in the southeast corner. The rainfall intensity of west section (WS) of the rainfall belt is weaker than the east section (ES).

As shown in Figure 2, all methods can produce similar pattern and magnitude of the precipitation with observations.
It is noted that DEA is more close to the observation in the WS. In the ES, the maximum rainfall in MRA1 was recorded by rain gauge more than $20 \mathrm{~mm}$ within one hour. KL, KFOI, and OI significantly overestimated the heavy precipitation in the MRA1. Other methods yielded similar precipitation estimation closed to the observation.

Compared with FEA, DEA could overcome the discontinuity of rainfall pattern in the east-south section. For the west section rain belt with weak precipitation, the maximum rainfall for one hour is recorded by gauge less than $20 \mathrm{~mm}$. The FEA produced heavy precipitation with intensity more than $20 \mathrm{~mm} / \mathrm{h}$. However, the DEA yielded much weaker precipitation less than $20 \mathrm{~mm} / \mathrm{h}$, which is close to the observation, indicating the promising performance of DEA in improving QPE.

In general, these 8 QPE methods (including 3 integrate methods) show the good performance for the event occurring at 17:00 to 18:00 (UCT) July 5, 2007, and all have some skills in estimating the precipitation. To quantitatively evaluate the estimation errors for all these QPEs, the relative errors $\left(\mathrm{RE}=\left(R_{r}(t)-R_{s}(t)\right) / R_{s}(t)\right)$ and correlation coefficients are analyzed for all rainfall patterns and strong intensity patterns ( $>10 \mathrm{~mm} / \mathrm{h}$ ) cases from June 30 to July 9, 2007. According to Table 2, the following can be seen:

(1) For all precipitation patterns, three integrated methods (KL, DEA, and FEA) show better performance with reduced relative errors and higher correlation coefficients than other methods.

(2) For the heavy precipitation region ( $>10 \mathrm{~mm} /$ hour), the KL and FEA are worse than AC. The DEA is more appropriate for QPE with reduced relative errors, and the correlation coefficient is equal to the maximum of $Z-R$ and four gauge correction methods. Generally speaking, DEA is the best and most effective method for precipitation estimation.

\section{Conclusion}

An integrated system for precipitation estimation was introduced in this paper. This system can effectively select the optimal QPE method from five different QPE methods for specific precipitation event by evaluating the variation of the random errors for different cloud types from latest 

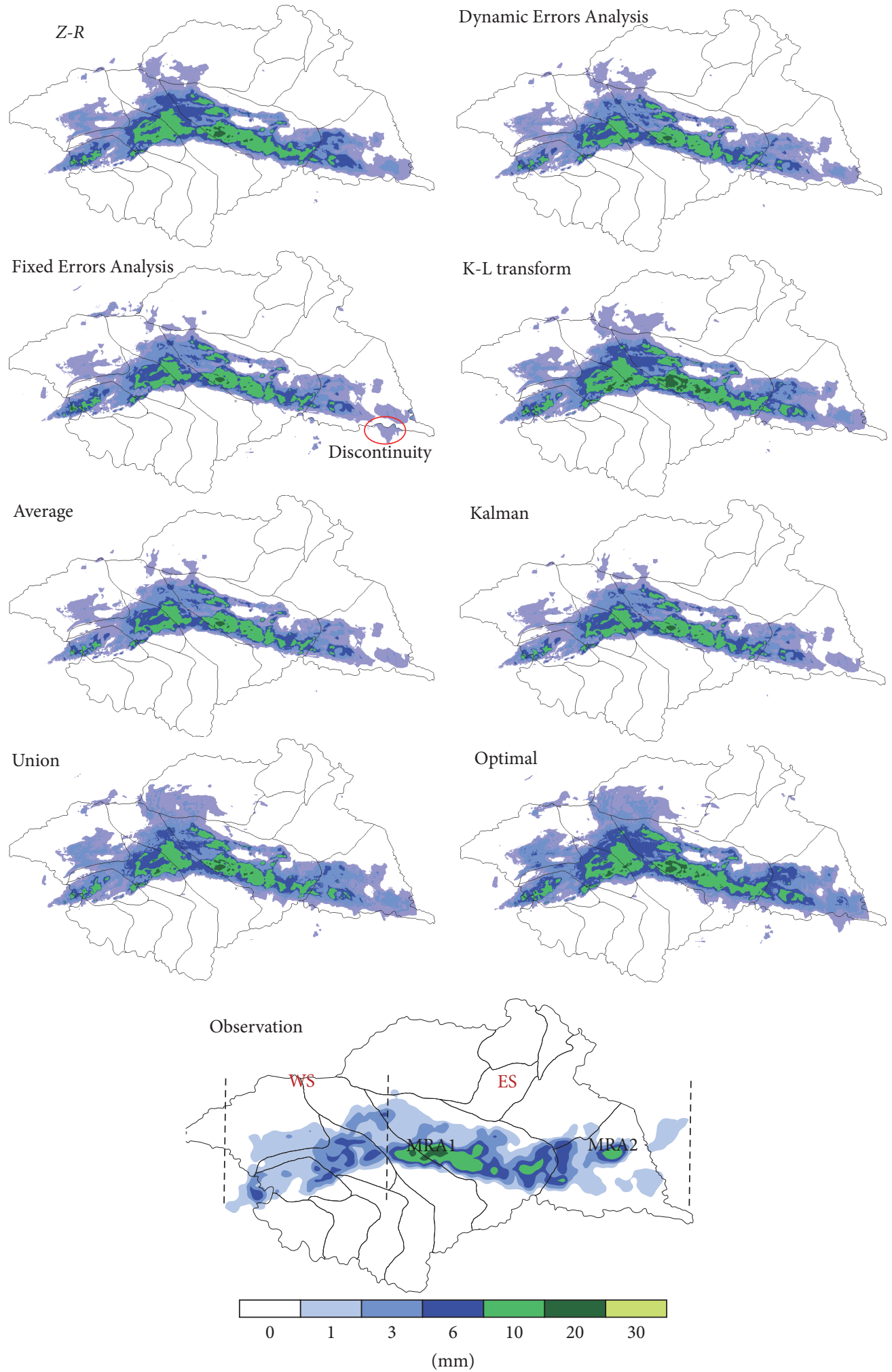

FIGURE 2: Rainfall distributions from 17:00 to 18:00 (UCT) July 5, 2007. 
precipitation estimates. The application of this integrated system on the event occurring in early July of 2007 shows that it can effectively determine the best estimation results from different QPE methods based on the capability of the stability of the precipitation estimation. This integrated method DEA with rainfall cloud classification could improve the application ability and precision of estimating precipitation for multiple precipitation types.

\section{Competing Interests}

The authors declare that they have no competing interests.

\section{Acknowledgments}

This study was supported by the National Natural Science Foundation of China under Grant no. 41275030, the R \& D Special Foundation for Public Welfare Industry (meteorology) of China under Grant no. GYHY201306040, the National Natural Science Foundation of Anhui Province under Grant no. 1508085MD64, and Shanxi Meteorological Research Foundation under Grant no. SXKKFRY20100101.

\section{References}

[1] J. Liu, Z. Song, D. F. Liu et al., "Classified Z-I relationship and its application to the measurement of rainfall by radar over the Huaihe River Basin," Scientia Meteorologica Sinica, vol. 19, no. 6, pp. 213-219, 1999 (Chinese).

[2] J. Pan and P. C. Zhang, "Estimating precipitation by radar measured vertical integration of liquid water," Journal of Nanjing Institute of Meteorology, vol. 23, no. 1, pp. 87-92, 2000 (Chinese).

[3] X.-Y. Liu, J.-T. Mao, J.-R. Li, and Y.-J. Zhu, "Application of radar-raingauge estimated mean area precipitation to runoff simulation," Journal of Hydraulic Engineering, vol. 22, no. 4, pp. 51-55, 2002.

[4] Y. Huang, W. Hu, A. Zhang et al., "Flood-causing torrential rain forecasting and warning system for Haihe River," Meteorological Monthly, vol. 32, no. 5, pp. 105-109, 2006 (Chinese).

[5] W. Zhenhui, P. Xiaofang, L. Jiantong, and G. Li, "Application of the principal characteristics extraction technique to consensus analysis of weather radar rainfall estimates," Inverse Problems, vol. 19, no. 3, pp. 525-531, 2003.

[6] L. Guan, Z. H. Wang, and X. F. Pei, "The consensus methods and effects of estimatiing rainfall using radar," Scientia Meteorologica Sinica, vol. 24, no. 1, pp. 104-111, 2004.

[7] J. S. Marshall, R. C. Langille, and W. M. Palmer, "Measurement of rainfall by radar," Journal of the Atmospheric Sciences, vol. 4, no. 4, pp. 186-192, 1947.

[8] L. Hu, Y. Zhao, Y. Liu, C. Scheepens, and A. Bouchard, "Updating multipoint simulations using the ensemble Kalman filter," Journal of Hydraulic Engineering, vol. 22, pp. 7-55, 2013.

[9] X. Liu, J. Mao, Y. Zhu, and J. Li, "Radar rainfall estimation and its application on runoff simulation over SHIGUANHE catchments," Acta Scientiarum Naturalium Universitatis Pekinensis, vol. 38, no. 3, pp. 342-349, 2002.

[10] E. A. Brandes, "Optimizing rainfall estimates with the aid of radar," Journal of Applied Meteorology, vol. 14, no. 7, pp. 13391345, 1975.
[11] J. Koistinen and T. Puhakka, "An improved spatial gage-radar adjustment technique," Bulletin of the American Meteorological Society, vol. 62, no. 6, p. 926, 1981.

[12] A. Rafieeinasab, A. Norouzi, D.-J. Seo, and B. Nelson, "Improving high-resolution quantitative precipitation estimation via fusion of multiple radar-based precipitation products," Journal of Hydrology, vol. 531, pp. 320-336, 2015.

[13] R. A. Fulton, J. P. Breidenbach, D.-J. Seo, D. A. Miller, and T. O'Bannon, “The WSR-88D rainfall algorithm," Weather and Forecasting, vol. 13, no. 2, pp. 377-395, 1998.

[14] WILSON JW, "Integration of radar and raingage data for improved rainfall measurement," Journal of Applied Meteorology, vol. 9, no. 3, pp. 489-498, 1970.

[15] R. Daley, Atmospheric Data Analysis, Combridge University Press, New York, NY, USA, 1991.

[16] Y. Wen, P. Kirstetter, Y. Hong et al., "Evaluation of a method to enhance real-time, ground radar-based rainfall estimates using climatological profiles of reflectivity from space," Journal of Hydrometeorology, vol. 17, no. 3, pp. 761-775, 2016.

[17] Q. Wei, Z. Q. Hu, L. P. Liu et al., "C-band polarization radar data preprocessing and its application to rainfall estimation," Plateau Meteorology, vol. 35, no. 1, pp. 231-243, 2016 (Chinese).

[18] Y. Huang, J. Ye, and G. Chen, "Consensus methods of estimating rainfall using radar base on error analysis," Journal of Atmospheric and Environmental Optics, vol. 5, no. 5, pp. 342-349, 2010.

[19] J.-Y. Ye, Y. Huang, Z.-J. Li, and Z.-B. Yu, "Rainfall estimation method based on multiple-doppler radar over the Huaihe river basin," Journal of Hydrologic Engineering, vol. 18, no. 11, pp. 14711476, 2013.

[20] Y. Huang, W. Hu, and H. Liu, "A method of cloud classification base on image entropy," in Proceedings of the 2nd International Congress on Image and Signal Processing (CISP '09), IEEE, Tianjin, China, October 2009.

[21] M. L. Baeck and J. A. Smith, "Rainfall estimation by the WSR88D for heavy rainfall events," Weather and Forecasting, vol. 13, no. 2, pp. 416-436, 1998.

[22] C. W. Ulbrich and L. G. Lee, "Rainfall measurement error by WSR-88D radars due to variations in $Z-R$ law parameters and the radar constant," Journal of Atmospheric and Oceanic Technology, vol. 16, no. 8, pp. 1017-1024, 1999.

[23] Z. Xiaohong, J. Tianshan, and H. Wen, "Multiscale circulation features of heavy rain over Huaihe River Basin in rainy season in 2007," Journal of Nanjing Institute of Meteorology, vol. 32, no. 2, pp. 321-326, 2009 (Chinese). 

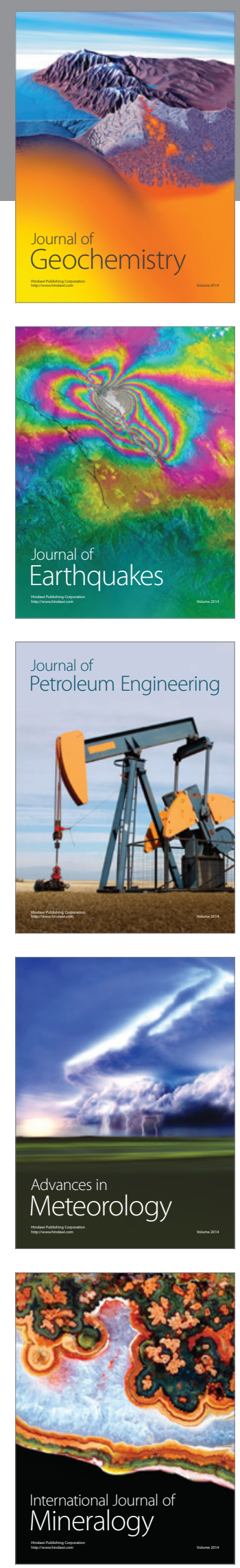
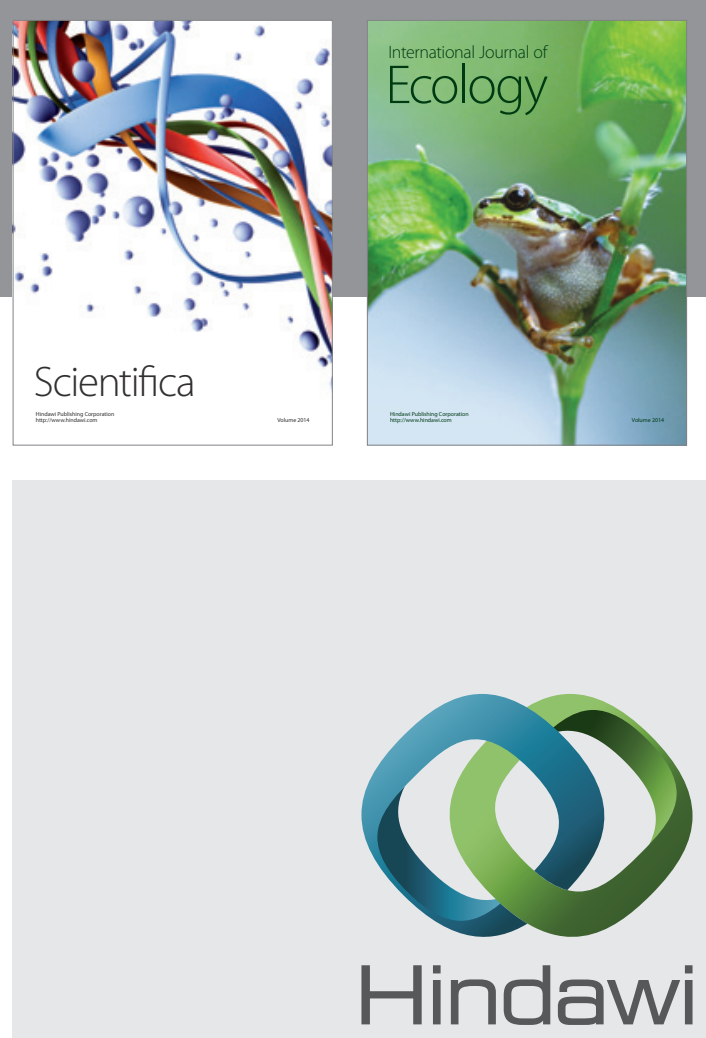

Submit your manuscripts at

https://www.hindawi.com
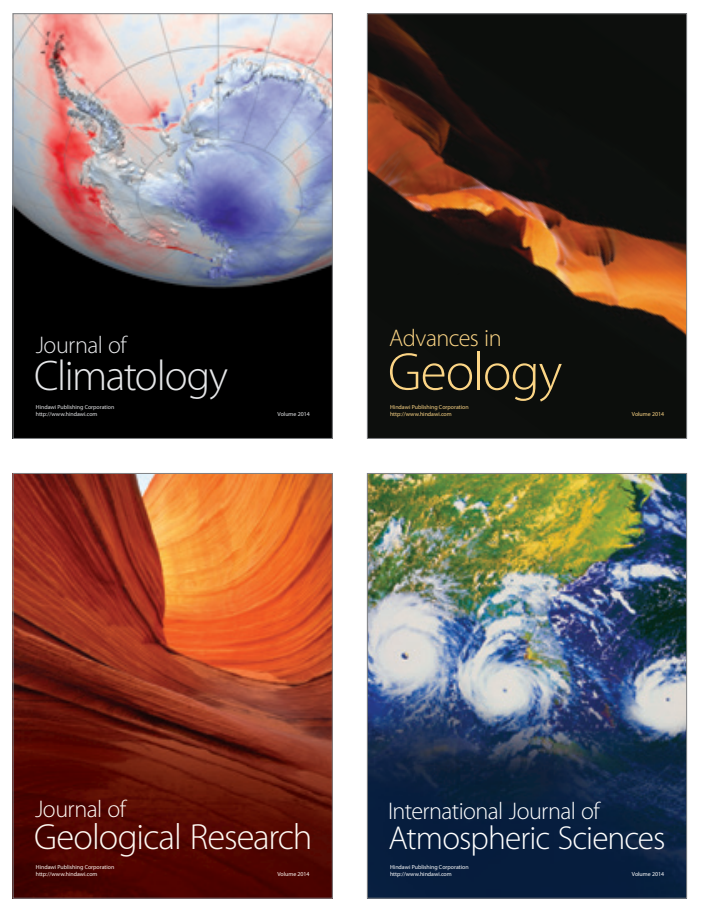

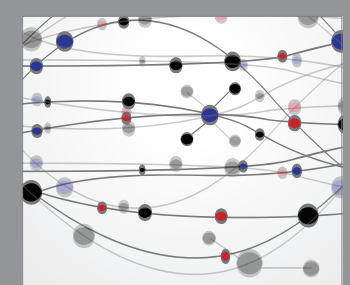

The Scientific

\section{World Journal}
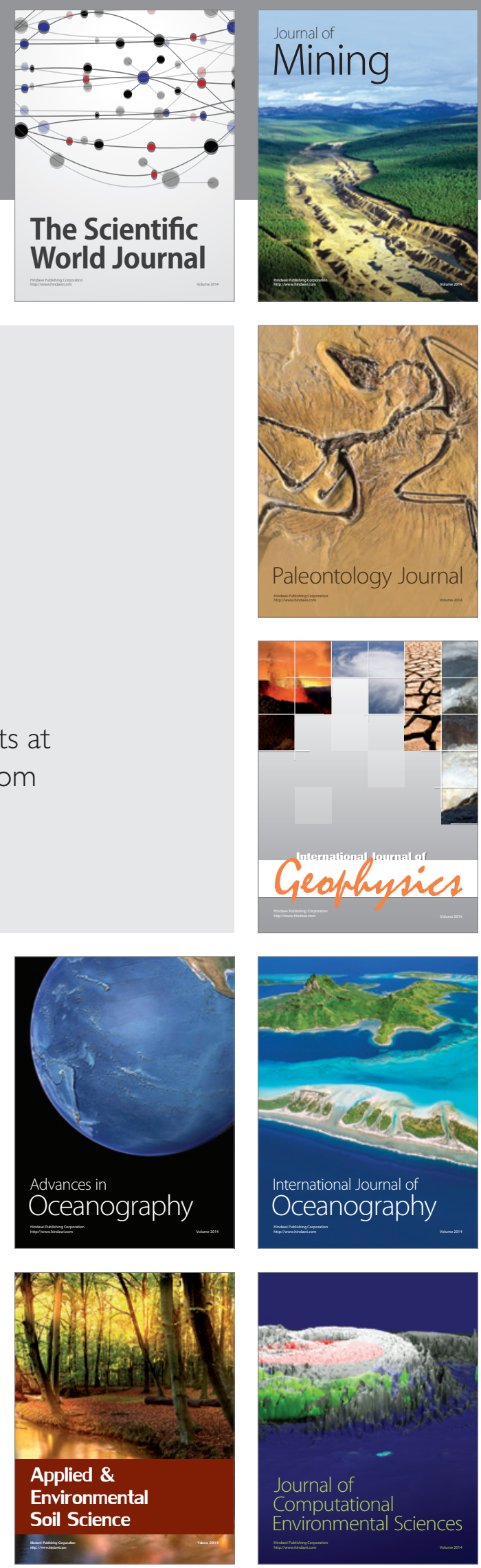